\title{
Modelling and Optimization of Bone-Cutting Forces in Orthopaedic Surgery
}

\author{
Christopher Plaskos ${ }^{1,2}$, Antony J. Hodgson ${ }^{2}$, and Philippe Cinquin ${ }^{1}$ \\ ${ }^{1}$ Laboratoire TIMC-IMAG, Groupe GMCAO \\ Faculté de Médecine de Grenoble \\ Joseph Fourier University, 38706 La Tronche, France \\ ${ }^{2}$ Neuromotor Control Laboratory, Department of Mechanical Engineering, \\ University of British Columbia, \\ 2324 Main Mall, Vancouver, B.C. Canada V6T $1 Z 4$ \\ \{plaskos, ahodgson\} @mech.ubc.ca
}

\begin{abstract}
Orthogonal cutting data is extremely useful since the measured force relationships can be used to model almost any machining process. However, previous orthogonal cutting investigations in bone have not tested conditions representative of clinical operations (i.e. very high tool speeds and small depths of cut, as in high-speed milling). In this paper, we aim to build a cutting force model that will be applied to the design, modeling, and optimization of a new robot-assisted high-speed milling system. Experimental results are presented for the cutting force components and the specific cutting energy of bovine cortical bone as a function of cutting tool geometry, depth of cut, and relative orientation between the cutting edge and bone structure. The cutting force magnitudes and trends prove to be considerably different from those previously reported in low-speed experiments, confirming the strong dependence of machining forces on cutting speed.
\end{abstract}

\section{Introduction}

Bone-cutting operations are of considerable importance in orthopaedic surgery. In total hip and knee replacement procedures, for instance, the geometrical accuracy of the prepared bone surface is particularly relevant to achieving accurate placement and good fixation of the implant. Recently, robotic guidance has been introduced into these procedures to improve bone-cutting precision, typically by rigidly attaching a high-speed milling tool to a large robotic arm. Although this may improve accuracy, many feel such instrumentation is not well suited to the operating room due to the size and safety issues; most existing systems use costly, industrial-sized robots that autonomously mill bone, giving the surgeon little control during cutting. Moreover, emerging less invasive techniques which require slender tools present even greater challenges and risks due to the reduced exposure of the bones.

A model of the cutting process would therefore be an invaluable tool not only for analysing stability and improving cutting performance in surgery, but also for de- 
signing compact and cost effective robotic guides and safer cutting techniques that directly involve the surgeon. In developing robotic systems, quantitative cutting force predictions would enable us to determine the strength and power requirements, the 'chatter' vibration characteristics, and the geometrical machining accuracy as a function of the mechanical stiffness and dynamic properties of the robot. A quantitative model would also allow us to simulate and optimize different surgical techniques and cutting tools, to analyse intra-operative force measurements, and to provide quantitative intra-operative guidance on key physical and safety parameters.

In this paper, orthogonal cutting is used as the basis for developing a mathematical model of the bone-cutting process. The force components tangential and normal to the cutting direction as a function of cutting tool geometry, depth of cut, and relative orientation between the cutting edge and bone structure (cutting mode) are presented for bovine cortical bone. In addition, the 'specific cutting energy' required to remove a unit volume of bone by cutting is presented for the above parameters.

Orthogonal cutting data has been previously acquired for very low cutting speeds $(\sim 10 \mathrm{~mm} / \mathrm{s}$ [1][2]), but since a 'softening effect' has been identified in bone when machining at higher speeds (see [4] for example), we need to re-evaluate the cutting properties of bone at clinically relevant cutting velocities (a speed of $3 \mathrm{~m} / \mathrm{s}$ corresponds to a rotational speed of 120,000 RPM for a cutting tool $3 \mathrm{~mm}$ in diameter). We also examine the role of parasitic or edge forces, which are present as the depth of cut approaches zero (typical of high-speed milling where chips are very thin).

\section{Methods}

\subsection{Workpiece Material - Bovine Bone}

Cortical bone specimens were harvested from the mid-diaphysis of fresh bovine femurs using an Exact diamond-blade band-saw under a continuous supply of irrigation. The cut specimens were potted into metal frames with dental cement and milled to a constant width of $\sim 5 \mathrm{~mm}$ and length of $\sim 40 \mathrm{~mm}$ on an industrial milling machine. The specimens were potted so that when they were clamped in the apparatus, the cutting direction was either transverse to, parallel to, or across the predominant bone fibre direction.

The structure in bovine cortical bone is relatively uniform and the direction of bone growth is readily discernible, as in human bone [5]. The microstructure of the specimens as seen in the plane transverse to the bone axis is shown in figure 1. Both primary laminar and secondary osteonal bone are present, and the anistropic structure of the bone is clearly evident. 

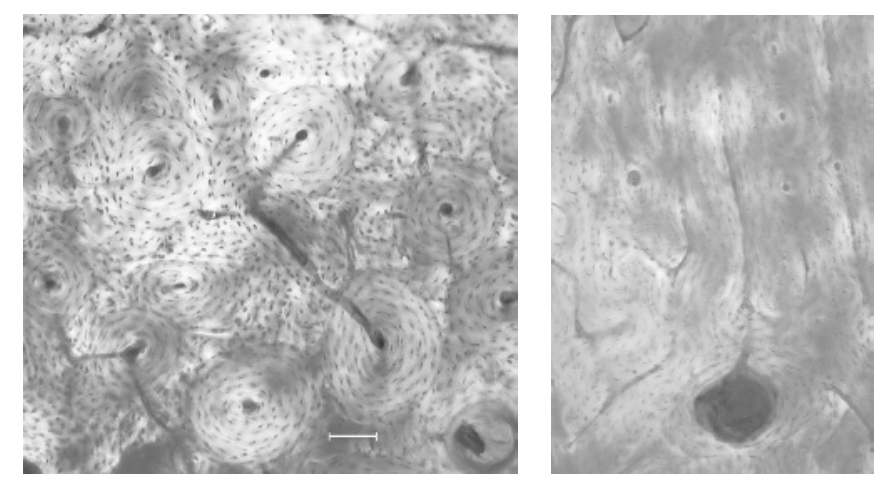

Fig. 1. Microscopic images showing the structure of the bovine cortical bone specimens in the transverse plane. Both osteonal (or Haversian, left image) and fibro-laminar (or plexiform, right image) structured bone is present. Scale bar represents $100 \mu \mathrm{m}$.

\subsection{Orthogonal Cutting Experiments}

Description of Variables. In the orthogonal cutting process, a single edge tool moves in a straight line with a velocity $v$ parallel to the surface of the workpiece, and the relationship between the force acting on the tool and the area of workpiece material being cut by the edge (uncut chip size) is examined (figure 2). The measured cutting force $(F)$ and the uncut chip area (normal to $v$ ) are normalized by the specimen width $(w)$, and the cutting force components per unit width $\left(F_{t}, F_{r}\right)$ are correlated to the depth of cut $(t)$.

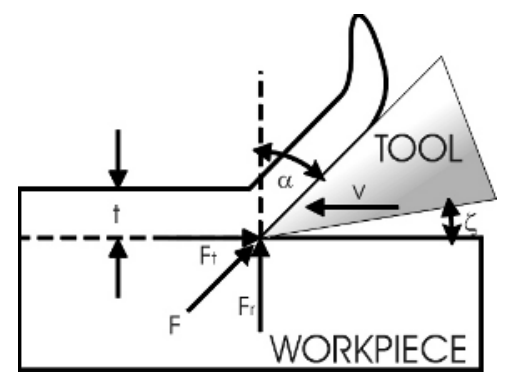

Fig. 2. Orthogonal Cutting Test: $F$ - resultant cutting force; $F_{t}-$ tangential cutting force; $F_{r}-$ normal (or radial) cutting force; $t$ - depth of cut or uncut chip thickness; $w$ - workpiece width into page; $\alpha$-rake angle; $\xi$-clearance angle; $v$-cutting tool velocity.

The 'specific cutting energy' represents the amount of work expended per unit volume of material removed in the cutting process, and it is defined by the input cutting power $\left(F_{t} \times v\right)$ divided by the material volume removal rate $(v \times w \times t)$ [3]. In orthogonal machining, this is simply equal to cutting force per unit cross-sectional area normal to the cutting direction: 


$$
E_{c}=F_{t} / t
$$

Experimental: In order to achieve high cutting-tool velocities $(v)$ and small depths of cut $(t)$ as encountered in high-speed milling operations, we used a specially designed pendulum apparatus. The device was equipped with a micron resolution dial gauge (Mitutoyo, $\pm 1 \mu \mathrm{m}$ ) which measured the tool advancement in the depth of cut direction. All experiments were conducted with a constant tool velocity of $3.5 \mathrm{~m} / \mathrm{s}$ (achieved by limiting the release height of the pendulum arm with a mechanical stop). The potted specimens were clamped on a piezoelectric dynamometer that measured cutting forces for tools with a range of positive rake angles $\left(\alpha=0,20\right.$, and $\left.40^{\circ}\right)$, depths of cut $(t=2-200 \mu \mathrm{m})$, and directions relative to the bone axis (transverse, parallel, and across the predominant bone fibre direction, figure 3 ). All cutting tools were precision ground from high-speed steel and had a clearance angle $(\xi)$ of $10^{\circ}$. The bone specimens were kept wet with physiologic saline throughout the cutting tests. Immediately after each cut, the formed chips were collected from a tray that lay beneath the apparatus and stored in labelled containers until microscopic analysis.

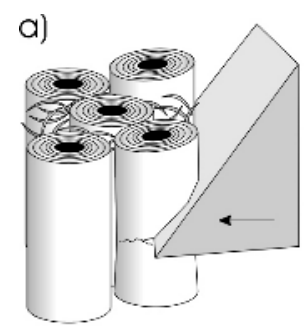

Transverse

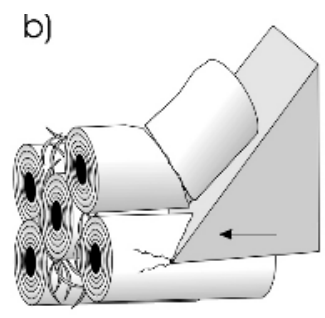

Parallel

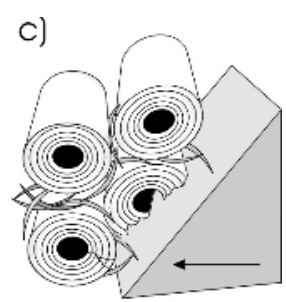

Across

Fig. 3. Illustrations of the cutting modes relative to the predominant osteon direction.

Data Collection: The piezoelectric force signals were amplified with charge amps (Kistler 5004 Dual mode amplifiers), low pass filtered at $200 \mathrm{~Hz}$ with a six order Butterworth analog filter (Krohn-Hite Corporation 3905B Multichannel Filter), and displayed on a four channel digital oscilloscope (Techtronics TDS 420A $200 \mathrm{Mhz} / 100 \mathrm{MS} / \mathrm{s})$. The scope was configured so that the filtered signals were superimposed directly onto the amplified force components. The unfiltered force signals generally exhibited some degree of fluctuation during the cutting process, where the proportional fluctuation in cutting force was generally more pronounced at larger depths of cut. To provide a repeatable measure of the average forces during cutting, the arithmetic mean of the filtered curve portion between the cut entry and exit points was used.

Data Analysis: The cutting force components per unit width tangential $\left(F_{t}\right)$ and normal $\left(F_{r}\right)$ to the cutting velocity are expressed as a function of depth of cut $(t)$ for each tool geometry and cutting orientation using the following power law [3]:

$$
F_{i}=K_{i c} t^{n_{i}}+F_{i e}
$$


where $n_{i}\left(0<n_{i}<1, i=t, r\right)$ are the constant parameters characterizing the 'size effect' of the particular workpiece material, and $K_{i c}$ are the constant parameters which characterize the local cutting mechanics and are dependent on the tool geometry and cutting direction relative to the bone anisotropy, and $F_{i e}$ are the residual force components per unit width at zero depth of cut (also referred to as edge or friction forces). It can be seen from Equation 2 that when the cutting force/depth of cut relationship is linear and there are no edge forces (i.e. $n=1$ and $F_{e}=0$ ), the specific cutting energy is constant and equal to $K_{c}$. However, when $n<1$ or $F_{e} \neq 0$ the energy required to remove a unit volume decreases as the depth of cut increases.

\section{Results}

The average tangential and radial cutting force components for cutting parallel to the fibre direction with three different tool geometries are plotted in figure 4. Similar results were obtained for the transverse and across cutting modes. Each point on the graph represents the average of four cuts, though the standard deviations (which were generally less than 5\%) are omitted for clarity.
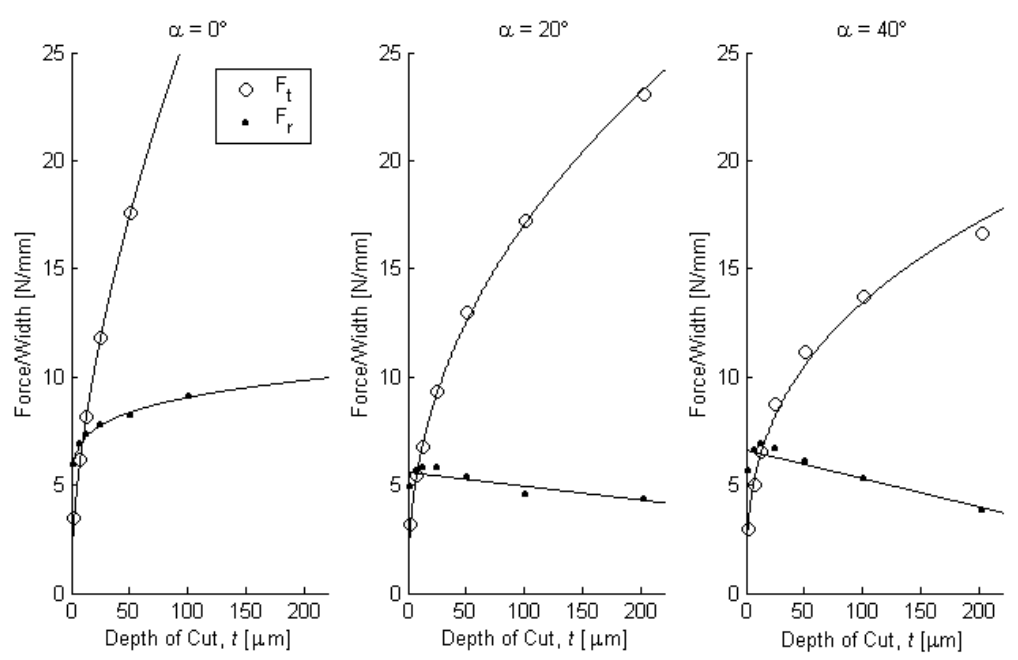

Fig. 4. Tangential $\left(\mathrm{O} F_{t}\right.$ ) and radial $\left(\cdot F_{r}\right)$ force components plotted against depth of cut for three different rake angles. The cutting direction is parallel to the predominant fibre direction

The general shape of the tangential force $\left(F_{t}\right)$ versus depth of cut $(t)$ curve for highspeed cutting is similar to those reported at low speeds [2], suggesting that the cutting force relationship is well modeled by the power law (equation 2) for a large range of cutting speeds (up to $3.5 \mathrm{~m} / \mathrm{s}$ ), depths (up to $0.2 \mathrm{~mm}$ ), and rake angles $\left(0-40^{\circ}\right.$ ). The magnitudes of the $F_{t}$ components are, however, generally only $\sim 30-50 \%$ of the low- 
speed values for all rake angles and cutting directions tested, indicating that the cutting coefficients $K$ and $n$ do exhibit a strong dependence on tool velocity.

Forces normal to the tool velocity $\left(F_{r}\right)$ exhibited a different relationship at high speeds when using highly raked tools $\left(\alpha=20-40^{\circ}\right)$, which suggests that the power law (equation 2) may not be a sufficiently accurate model for the radial force. For all fibre orientations, $F_{r}$ tended to increase initially until it reached a maximum value at a cutting depth of $t \sim 15 \mu \mathrm{m}$, and then it decreased as the depth of cut increased. The rate of decrease was generally more drastic at higher rake angles. At low speeds, $F_{r}$ has been reported to increase slightly with cutting depth even for positively raked tools [1][2].

The tool geometry also had a significant effect on the cutting coefficients, where $K_{c}$ decreased dramatically from $\sim 65$ to $18 \mathrm{~N} / \mathrm{mm}^{2}$ as the rake angle increased from $0^{\circ}$ to $40^{\circ}$ for all cutting directions. The average ratios, $F_{t, \alpha=40^{\circ}} / F_{t, \alpha=0^{\circ}}$ and $F_{r, \alpha=40^{\circ}} / F_{r, \alpha=0^{\circ}}$, in the $50-200 \mu \mathrm{m}$ depth of cut range are $\sim 0.48$ and 0.33 respectively, which indicates the improvement in cutting efficiency with positive rake angle. As the depth of cut approaches zero, however, the measured tangential and radial forces (i.e. at $t \sim 2 \mu \mathrm{m}$ ) tended to constant values of $\sim 3.8$ and $\sim 5.5 \mathrm{~N} / \mathrm{mm}$ respectively, regardless of the tool rake angle. It is likely that these parasitic forces are primarily due to friction acting between the bone and the clearance face, which was $10^{\circ}$ for all tools. A constant $F_{t} / F_{r}$ ratio across all cutting modes at zero depth of cut suggests that a simple coulomb friction law $\left(\mu=F_{l} / F_{r} \sim 0.7\right.$ ) would be adequate to describe the physical process acting on the clearance face of the tool. Here, the radial force component $\left(F_{r}\right)$ is primarily a result of the bone being compressed under the tool clearance face (i.e. material 'spring-back'), and the tangential force $\left(F_{t}=\mu F_{r}\right)$ is due to the friction that acts on this face as the tool slides over the bone. These force values were only slightly affected by fibre orientation, exhibiting a variation of $\sim 10 \%$ across all directions, which suggests that the coefficient of friction between the bone and steel is largely independent of fibre orientation.
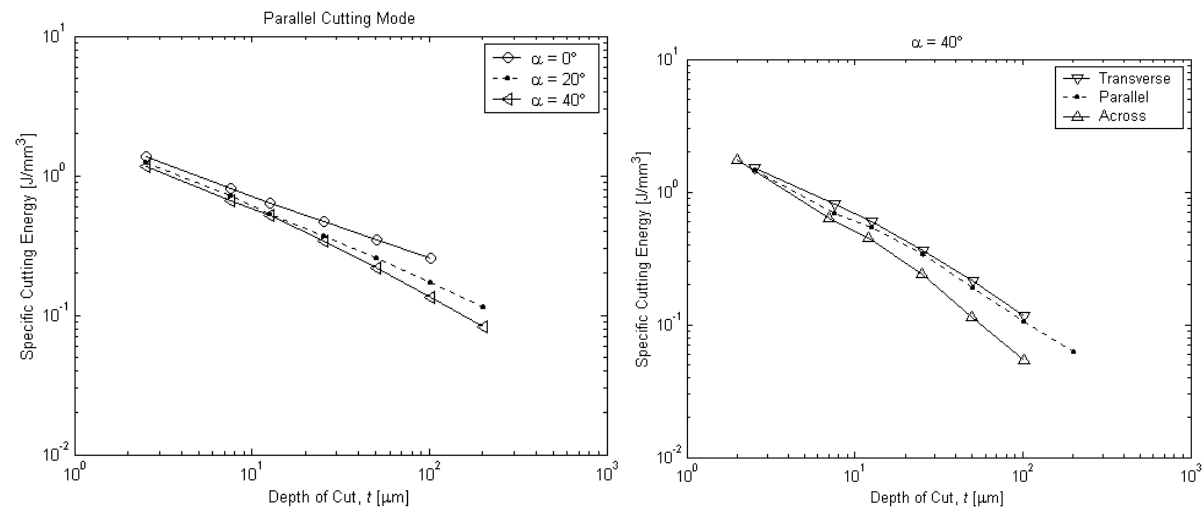

Fig. 5. Specific cutting energy vs. depth of cut plotted on log-log coordinates. Left: three rake angles and one cutting mode (parallel). Right: three cutting modes and one tool angle $\left(\alpha=40^{\circ}\right)$. 
The relationships between the specific cutting energy and depth of cut for three different rake angles and three different cutting depths is shown on log-log coordinates in figure 5. Similar to low-speed data, the specific energy values fall close to straight lines when plotted in this manner, though the slopes and positions (i.e. the energy magnitudes) of the lines are different [2]. It can be seen that as the depth of cut increases, the amount of work required to machine a unit volume of bone decreases for all tool geometries and cutting modes. This decrease in specific cutting energy with depth of cut is due to the nonlinear increase in the tangential cutting force component as seen in figure 4.

Observations on Chip Formation: The chip formation mechanism at small depths of cut tended to resemble the classical 'shear plane' process commonly seen in metal cutting, where chip segments were more or less continuous structures which slid up the tool rake face, curling as the tool progressed through the workpiece (figure 5). This differs from the chip fracture mechanism previously observed for small depths of cuts in low-speed orthogonal cutting [1][2]. However, our chips are similar to those observed in bone-drilling operations [4][5], which suggests that the high-speed orthogonal cutting mechanism is more representative of actual surgical operations.

As the depths of cut was increased, the chip formation mechanism began to change to one of discrete fracture, where the chips tended to appear undeformed and segmented in nature. The degree of subsurface damage below the cutting plane increased significantly as $t$ approached $200 \mu \mathrm{m}$. This was particularly evident from the lower rake angles and in the transverse cutting mode (i.e. when forces were largest), as gaping craters and cracks were observed on the cut surface. This cutting condition was characterized by large, erratic fluctuations in the unfiltered force signal, which was likely a result of the fracture process. However, cutting depths this large are not typically encountered in surgical operations when using rotary power tools.
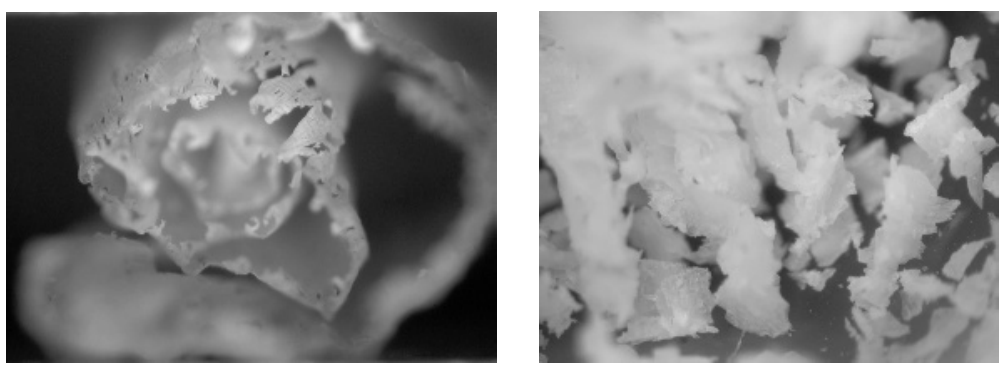

Fig. 6. Macroscopic images illustrating different chip formation mechanisms for $2 \mu \mathrm{m}$ (left) and $200 \mu \mathrm{m}$ (right) depths of cut.

\section{Discussion}

Despite a considerable amount of research and development of robotic assisted tools for bone-cutting operations, few quantitative analyses of the cutting process exist and 
surgical variables often seem to be chosen arbitrarily. Previous related work has mainly focused on bone-drilling [4][5]. However, one major drawback to approaches adopted in these studies is that the measured relationships are generally only valid for the particular drill bit geometry used in the study. Orthogonal machining on the other hand, is the simplest case of cutting and is fundamental to almost all other cutting operations, such as milling, drilling, and sawing [6]. Quantifying the relationships between cutting force, depth, mode, velocity and tooth geometry will allow us to simulate surgical cutting operations for tools of any shape, operating at any feed rate, speed, depth and so on [7].

Application to Force Prediction in Bone-Milling: We are particularly interested in maximizing the accuracy of planar bone cuts made in total knee arthroplasty and have found empirically that a side-milling tool cuts much more accurately than a bone saw. As an example of how we would make use of the data from this study, consider the problem of optimizing the design of the side-milling system. The surgeon would need to understand how to trade off cut width against feedrate to minimize the time spent making the cut. Furthermore, the surgeon would need to be able to predict how much the tool will deflect due to the cutting forces in order to control the accuracy of the cut.

Future work in our laboratory will include investigating the cutting mechanics of cancellous bone in the knee, before conducting a series of bone-milling experiments in which we compare measured milling forces to our predicted ones.

\section{Conclusions}

Bone cutting forces are strongly dependent on tool velocity, and although low speed cutting studies may be useful for examining general bone-cutting mechanics, such data is not directly applicable to modelling high-speed milling. The new data presented is thus invaluable to the developers of CAOS cutting systems.

\section{References}

1. Jacobs CH, Pope MH, Berry JT, Hoaglund F: A study of the bone machining process Orthogonal cutting. J. Biomechanics. (1974) 7: 131-136

2. Wiggins KL, Malkin S. Orthogonal machining of bone. J. Biomech. Eng 1978 (100) 122 130

3. Oxley PLB. Mechanics of machining: an analytical approach to assessing machinability. Ellis Horwood Ltd. Chichester England. 1989

4. Jacobs CH, Pope MH, Berry JT, Hoaglund F. A study of the bone machining process Drilling. J. Biomechanics. (1976) 9: 343-349

5. Wiggins KL, Malkin S. Drilling of Bone. J. Biomechanics. (1976) 9: 553-559.

6. Krause WR. Orthogonal Bone Cutting: Saw design and Operating Characteristics. J. Biomech. Eng. (1987) 109: 263-271

7. Budak E, Altintas Y. Prediction of milling force coefficients from orthogonal cutting data. Manufacturing Science and Engineering (1993) 64: 453-460 